https:/ / creativecommons.org/ licenses/ by/ 4.0/

\title{
PROPUESTA PARA LA ESTANDARIZACIÓN DE PROCESOS EN EL ÁREA DE CALIDAD, SEGURIDAD DEL PACIENTE Y SARLAFT (CASO ENTIDAD ONCOLÓGICA COLOMBIANA) ${ }^{1}$
}

\author{
Proposal for the standardization of processes in the area of quality, \\ patient safety and SARLAFT (C ase of colombian oncological entity)
}

\author{
STEEVEN R. SALCED O 2, WILMER A. ROA3 , EVER A. FUENTES 4 \\ Recibido:01 de febrero de 2020. A ceptado:13 de febrero de 2020 \\ D OI: http://dx.doi.org/10.21017/rimci.2020.v7.n14.a83
}

\begin{abstract}
Resumen
La falta de procesos estandarizados, puede convertirse en un serio problema dentro de las organizaciones, ya que podría atra er consecuencias como: la existencia de no conformidades en los productos y/ o servicios, la insatisfacción del cliente, sobrecarga de trabajo, entre otros. El área de Calidad, Seguridad del paciente y SARLAFT de la entidad O ncológica colombiana, objeto de este trabajo, se ha visto afectada por esta problemática, debido a esto y buscando una solución a la misma se desarrolló este proyecto.

En la entidad oncológica, se pretende abarcar el problema de la no estandarización, comenzando con un diagnóstico de entrada para comprender el estado de los procesos de la institución, seguido de una adecuada documentación y toma de tiempos de los procedimientos, para finalizar con la generación de indicadores de control y la medición de los costos de no calidad del sistema.

Luego de haber aplicado la estandarización, se obtuvo el tiempo idóneo para cada uno de las actividades realizadas por el área; además, se aplicó la metodología PEF (Modelo de Prevención, Evaluación y Fallos), para el análisis de los costos de Calidad y No Calidad en la institución.

Con los resultados obtenidos de esta investigación, se logró hacer una propuesta a la entidad o ncológica colombiana, en la cual se hizo la identificación de los procesos que no generan valor agregado a las actividades del área y también de aquellos que presentan un riesgo elevado para el funcionamiento de la institución.
\end{abstract}

Palabras clave. Calidad, Estandarización, Proceso, SARLAFT.

\begin{abstract}
The lack of standardized processes can become a serious problem within organizations, as it could attract consequences such as: the existence of non-conformities in products and / or services, custo mer dissatisfaction, work overload, among others. The area of Q uality, Patient Safety and SARLAFT of the C olombian O ncology entity, object of this work, has been affected by this problem, due to this and looking for a solution to it, this project was developed.

In the oncological entity, it is intended to cover the problem of non-standardization, starting with an initial diagnosis to understand the state of the institution's processes, followed by adequate documentation and time-taking of the procedures, to end with the generation of control indicators and the measurement of non-quality costs of the system.
\end{abstract}

1 A rtículo desarrollado del proyecto < < Propuesta para la estandarización de procesos en una entidad oncológica colombiana > >, ejecutado entre octubre de 2018 y enero de 2020 desde el programa de Ingeniería Industrial de la Universidad Libre, sede Bogotá

2 Ingeniería Industrial, Estudiante de la Facultad de Ingeniería, Universidad Libre Bogotá - Colombia. O RCID: https://orcid.org/0000-00025179-2998

3 Ingeniería Industrial, Estudiante de la Facultad de Ingeniería, U niversidad Libre Bogotá - Colombia. O RCID: https://orcid.org/0000-00019304-7681

4 M BA., profesor de la Facultad de Ingeniería, Universidad Libre Bogotá - Colombia ID: https://orcid.org/0000-0001-9671-5884 Correo Electrónico: ever.fuentes@unilibre.edu.co

Rev. Ingeniería, M atemáticas y Ciencias de la Información

Vol. 7 / Núm. 14 / julio - diciembre de 2020; pág. 39-57 
After having applied the standardization, the ideal time was obtained for each of the activities carried out by the area; Furthermore, the PEF methodology (Prevention, Evaluation and Failure Model) was applied to analyze the costs of $\mathrm{Q}$ uality and $\mathrm{N}$ on-Q uality in the institution.

With the results obtained from this research, it was possible to make a proposal to the Colombian 0 ncology entity, in which the identification of the processes that do not generate added value to the activities of the area and also of those that present a high risk for the operation of the institution.

Key words. Q uality, Standardization, Processe, SARLAFT.

\section{INTRODUCCIÓN}

NO DE los temas demayor complejidad delos últimos tiempos está relacionado con las condiciones de las instituciones que trabajan en salud. Se gún la Organización Panamericana paralaSalud [1], en un mundo globalizado como el de hoy, los gobiernos no han conseguido responder a las demandas de la población en lograr mejores servicios sanitarios y por lo tanto una mejor calidad de vida.

Según Donabedian [2] la percepción de la calidad en la atención médica es una idea sumamente difícil de definir, debido a su rigurosidad técnicocientífica con la quese debeasumir, cómo el significado práctico quetienela percepción del usuario para cada uno [3].

En la entidad oncológica colombiana, ubicada en la ciudad de Bogotá, los procesos del área "Calidad, Seguridad del Pacientey SARLAFT", seencuentran desactualizados y es necesario el desarrollo de operaciones estandarizadas, con el objeto de evitar la discrepancia entre las actividades realizadas.

Para dar solución a esteproblema, se realizó un trabajo de campo en la institución que consistió en aplicar un diagnóstico de entrada al estado de los procesos. Posteriormente, con los resultados obtenidos, se desarrollaron y actualizaron los documentos que requerían de ello. Se aplicó una metodología de toma de tiempos, se calculó el estándar para cada una delas actividades del área.

Posteriormente, seestablecieron indicadores de gestión y seaplicó un model o para cal cular los costos de calidad y no calidad. Con el desarrollo de este proyecto, se espera quela entidad oncológica colombiana, disponga deunos procesos documentados y estandarizados para el área, con el objetivo de que pueda identificar aquellas actividades que no generan valor agregado a su cadena de valor.

\section{Antecedentes}

El presente trabajo, aborda una exhaustiva re visión literaria de los tres pilares fundamentales, objeto de la estandarización de procesos del presente trabajo, quienes son: Calidad, Seguridad del Pacientey SA RLAFT; área dela entidad oncológica ubicada en la ciudad e Bogotá

Inicialmente, con objeto de ahondar en la me todología SA RLAFT, se consultó la investigación de Nuñez, Mora \& Á vila [4] la cual consistió, en la implementación de un sistema contra el lavado de activos en los agentes de seguridad social en salud.

El trabajo aborda tres conceptos notables, básicos para la implementación del SARLAFT en las entidades de salud, los cuales son:

- Lavado de Activos (LA): Es el mecanismo que busca dar apariencia de legalidad a los dineros que provienen deactividadesilícitas como el narcotráfico, corrupción, extorsión, entre otros.

- Financiación del Terrorismo (FT): Se basa en destinar dinero lícito o ilícito a la financiación deactividades consideradas terroristasy queson real izadas por gruposal margen de la ley.

- Unidad de Investigación y A nálisis Financiero (UIA F): Es quién vigila las actividades inusuales o sospechosas de las organizaciones, responsable deimplementar Sistemas de Administración del Riesgo de Lavado de Activos y Financiación del Terrorismo (SARLAFT)

Esta investigación se tomó en cuenta, porque comparte directrices y los conceptos clave, que se deben tener en cuenta dentro del sistemaSARLAFT y también trata la metodología que se debe seguir

Rev. Ingeniería, M atemáticas y Ciencias de la Información Vol. 7 / Núm. 14 / julio - diciembre de 2020; pág. 39-57 
para el buen funcionamiento deestesistema y para la prevención de estetipo deriesgos en las entidades de salud.

El segundo tema a tratar, para el análisis de la presente investigación está relacionado con la estandarización de procesos. Para desarrollar ese asunto, seseleccionó el trabajo realizado por Pérez [5] en una empresa de textiles, el cual consistió en documentar y estandarizar las actividades productivas dela compañía, para disminuir desperdicios de materia prima y definir con exactitud las labores de los operarios.

La problemática de la compañía estaba dividida entre la falta de organización en sus procesos y documentación respectiva delas actividades. A unque los trabajadores tenían experiencia en la elaboración de los tejidos, no presentaban un orden en la elaboración de sus productos, cuyo efecto repercutía en insatisfacción del cliente y ausencia de calidad.

Para solucionar dichos problemas el autor planteó una metodología que consistió inicial mente, en realizar un diagnóstico de entrada para comprender el estado de los procesos de la compañía, donde aplicó una matriz para el análisis de factores. Posteriormente, se siguieron los siguientes pasos, para solucionar el problema dela estandarización:

- Desarrollar formatos para acciones preventivas, correctivas y acciones.

- Desarrollar los procedimientos einstructivos.

- Desarrollar procedimientos y registros para auditorías internas.

- Elaborar el control dedocumentos, registros y producto no conforme.

- Elaborar las caracterizaciones delos procesos.

- Elaborar listas maestras de documentos y registros.

- Levantamiento de procesos y mapa de procesos.

- Realizar los documentos y registros necesarios para los procesos.
Los resultados obtenidos, después de haber aplicado el proyecto fueron la obtención del Sistema de Gestión de Calidad ISO 9001:2008, vigente para ese entonces y los procesos de la planta de producción estandarizados cuyo impacto fue la disminución del desperdicio y la pérdida de insumos, tales como materia prima y tiempo; recursos con los que se logró aumentar la productividad y por ende las utilidades de la empresa.

Este trabajo sirve como base para la presente investigación, ya que aporta, fundamentos, metodología y procedimientos clave para la estandarización de procesos dentro de una compañía. Cabe resaltar que el sector al que se aplicó dicho trabajo no es el mismo de esta investigación, pero dichos principios aplican como soporte para este proyecto.

Para efectos de abordar un conocimiento profundo acerca de los procesos de seguridad del paciente, se examinó la investigación realizada por Franco [6], donde el autor pretende explicar la dimensión del problema existente alrededor de la atención en salud.

Para lograr un mayor entendimiento del problema, el autor propone conocer la siguiente terminología relacionada al tema:

- Evento adverso: Hace referencia al resultado clínico que es adverso al esperado y debido a error durante el diagnóstico, tratamiento o cuidado del paciente y no al curso natural dela enfermedad o a condiciones propias del mismo. Por tanto, aquel evento adverso que hubiera sido posible prevenir utilizando medidas razonables, es por definición un error clínico.

- Seguridad clínica y calidad de la atención: La seguridad clínica forma parte integral de la dimensión técnica de la calidad de atención. El decreto 2309/ 02, reglamentó la calidad de atención en salud en Colombia y consideró, junto con el acceso a los servicios de salud, con la oportunidad en la atención, con la pertinencia del enfoque dínico, con la continuidad de la atención y con la seguridad del pacientecomo las característicasfundamentales de la calidad de la atención y obliga por ley a las instituciones de salud a lograrlas. 
- Cultura de seguridad y prácticas seguras: Por cultura organizacional seentiendeel conjunto de creencias, valores y actitudes que comparten los miembros de una organización y que sereflejan en sus acciones diarias.

También se observó que los principales pasos, para la seguridad de la atención en salud son:

- Establecer un sistema de reporte de eventos adversos.

- Establecer un sistema de auditoría de resultados clínicos no esperados.

- Establecer un sistema de mejoramiento continuo de la calidad tendiente a disminuir la incidencia de eventos adversos.

El autor destaca en su investigación, que la seguridad del pacientees un tema detodos los agentes involucrados en el sector salud, desde las instituciones deeducación universitarias hasta los entes gubernamentales, no es sól o tema de los hospitales de los países desarrollados. También resalta de la importancia de generar una cultura en seguridad del paciente, para minimizar el riesgo del error clínico.

La investigación mencionada previamente, se tomó como referencia de este trabajo, ya que el área de estudio está comprendida por (Calidad, Seguridad del Pacientey SARLAFT) y con estetrabajo se buscaba comprender el marco lógico de la seguridad del paciente, con el propósito de profundizar en los conceptos técnicos y los fundamentos de dicho tema.

\section{Metodología}

La metodología escogida para el desarrollo de la presente investigación, está compuesta por cinco etapas:

\section{A. D iagnóstico}

Inicialmente, se hizo una revisión literaria sobre metodologías aplicables al proyecto, cuyo resultado fuera, un diagnóstico de entrada, que arrojara el estado de los procesos y los problemas primordiales de la entidad.
Para tal fin, se aplicaron las siguientes cuatro herramientas:

- M atriz D OFA : Según Thompson [7], “El análisis FODA consiste en realizar una evaluación de los factores fuertes y débiles que, en su conjunto, diagnostican la situación interna deuna organización, así como su evaluación externa, es decir, las oportunidades y amenazas".

- M atriz V ester: Según Betancourt [8], es una matriz, compuesta por filas y columnas, en dónde se representan las posibles causas de una situación. Además, se puede caracterizar esta herramienta como una metodología de marco lógico, es decir, aquellos instrumentosquepermiten capturar lainformación, plantear, caracterizar y priorizar problemas, entre otros

- Encuesta: El autor García Ferrando [9], definela encuesta es una investigación realizada sobre una muestra de sujetos representativa de un colectivo más amplio, que se lleva a cabo en el contexto de la vida cotidiana, utilizando procedimientos estandarizados de interrogación.

- D iagrama Causa - Efecto: Según Ishikawa [10], "Los diagramas Causa-Efecto ayudan alos estudiantes a pensar sobretodas las causas reales y potenciales de un suceso o problema, y no solamente en las más obvias o simples."

Después de aplicadas las herramientas citadas anteriormente, se determinó el panorama interno y externo de la entidad oncológica, con sus causas y posibles fallos asociados.

\section{B. Documentación}

Una vez aplicadas las herramientas diagnósticas, seobtuvo el contexto lógico dela entidad y seprocedió a realizar el respectiva ajustey desarrollo de documentación de los procesos realizados dentro del área Calidad, Seguridad del Paciente y SARLAFT.

Para el desarrollo de este punto, se utilizó como base el trabajo realizado por Pérez [5], en el cuál 
hicieron una estandarización deprocesos, dóndetuvieron que levantar procesos, acoplar otros, realizar lascaracterizaciones deactividades, losmanuales de calidad y diagramas de procesos, entre otros.

Inicialmente, se elaboró un análisis sobre el estado de la documentación, donde se identificaron las necesi dades en estetema quepresentaba el área. Seguido a esto, se actualizó la caracterización del área, los procesos y formatos que se encontraban desajustados y secrearon losinstructivos, formatos y documentos que hacían falta.

\section{Tiempos y estandarización}

Para la medición del trabajo en la entidad oncológica colombiana, en un principio, seclasificaron las actividades del área en dos categorías: Actividades rutinarias y actividades no rutinarias.

La toma de tiempo de los procesos rutinarios, se llevó a cabo utilizando la metodología descrita por Criollo \& Magaña [11], dónde se aplicó el método de fórmulas estadísticas para lograr el cálculo del tiempo estándar.

Este método consiste en el cál culo del número de observaciones a través de unas tomas de tiempo preliminares con un porcentaje de riesgo determinado. Se aplican las siguientes fórmulas (Ver Fig. 1 y 2):

$$
N=\frac{K * \sigma}{e *{ }^{-} \boldsymbol{x}}
$$

Fig. 1. Fórmula frecuencia [11].

$$
\sigma=\sqrt{\frac{\sum \int(X i-\bar{x})^{2}}{n}}
$$

Fig. 2. Fórmula desviación [11].

Dónde:

-x: Media aritmética de los tiempos

n: Número de mediciones efectuadas

e: Error expresado en forma decima

Xi: Valores obtenidos de los tiempos de reloj

$\mathbf{N}$ : Frecuencia de cada tiempo

K: Coeficiente de riesgo cuyos valores son:

K: 1 para riesgo de error $32 \%$

K: 2 para riesgo de error $5 \%$

K: 3 para riesgo de error $0.3 \%$
Para la medición de las actividades no rutinarias, se tomó como base la metodología utilizada en la guía metodológica para el estudio de cargas de trabajo Universidad Nacional [12], donde se tomaron tres expertos relacionados con los procesos dela entidad oncológica, los cuáles fueron entrevistados para conocer el tiempo estándar de dichas operaciones.

\section{Indicadores}

Setomó como basela metodología KPI, expuesta en el trabajo de Velimirovi?a, Velimirovi?b, Stankovi?a [13], que describe la importancia de la aplicación delos indicadores degestión o Key Performance Indication dentro de cualquier sector productivo de la sociedad.

Por lo anterior seestableció que cada indicador debía responder 6 preguntas de manera específica, estas son: ¿Qué?, ¿Dónde?, ¿Cómo?, ¿Cuándo?, ¿Quién?, ¿Por qué?, después se actualizó la ficha técnica individual por cada uno, donde era necesario definir: el objetivo, fórmula, unidad de medida, nombre de las variables, fuente de información, periodicidad de informe, reporte, partes interesadas y tipo deindicador (eficiencia, eficacia o efectividad).

Finalmente, se hizo una asignación de responsabilidades, tanto de recolección de datos como de análisis y reporte. A dicional a esto, se establecieron en la ficha los campos para realizar el seguimiento a estos datos, donde se puede evidenciar su comportamiento tanto numérica como gráficamente.

\section{E. A nálisis de costos}

Finalmente, y con el propósito de darle un valor agregado a la investigación realizada, se consultó una metodología que se ajustara a los parámetros de la entidad oncológica y diera como resultados el cálculo de los costos de calidad y no calidad.

La metodología utilizada, consistió en la aplicación del modelo PEF (Prevención, Evaluación y Fallos) [14] los costos de calidad son aquellos incurridos en la mejora de los sistemas de las empresas, también los asociados a fallos del sistema, producto y servicio. 
Para la aplicación de dicha metodología dentro dela entidad oncológica de estudio, setomó como referencia, el trabajo realizado por Tapia y Rodolfo [15] el cuál consistió en la aplicación del modelo Prevención, Evaluación y Fallos, en una empresa del sector textil.

El modelo, se basó en los siguientes pasos:

- Recolectar y cuantificar los costos.

- Calcular los costos de prevención, evaluación y fallos.

- A nalizar y detectar mejoras.

- A nálisis delos costos de calidad totalesy no calidad totales.

- Detección de procesos por mejorar y oportunidades de mejora.

\section{Resultados}

\section{A. Diagnóstico}

Para realizar el anál isis de entrada de la institución, inicialmenteseaplicó la matriz DOFA, con la que se identificaron las principales fortalezas y debilidades tanto internas como externas de la compañía.

La información se recolectó, con entrevistas al personal profesional de Calidad, quién orientó el panorama actual dela gestión documental, delos procesos, de los riesgos, peligros y oportunidades de mejora que hay en las áreas deCalidad, Seguridad del Paciente y SARLAFT.

Con los problemas obtenidos del análisis DOFA, se construyó una matriz de Vester (Ver tabla 1), con la cual se buscó priorizar los problemas encontrados y así mismo, realizar un análisis de las causas de la problemática encontrada.

Se puede observar de la matriz (Ver tabla I), que los tres problemas que tienen más influencia (Total activos) sobre los otros son:

1. Desactualización delos documentos.

2. Sobrecargo de responsabilidades.

3. Poca confiabilidad en el software existente.

También, seidentificaron los tres problemas que más dependencia (Total pasivos) tienen sobre los demás:

Tabla. I. Matriz de Vester.

\begin{tabular}{|c|c|c|c|c|c|c|c|c|c|}
\hline Número & Descripción de los problemas & 1 & 2 & 3 & 4 & 5 & 6 & 7 & $\begin{array}{c}\text { Total } \\
\text { Activos }\end{array}$ \\
\hline 1 & $\begin{array}{l}\text { Desactualización de los } \\
\text { documentos }\end{array}$ & 0 & 3 & 2 & 1 & 3 & 2 & 3 & 14 \\
\hline 2 & $\begin{array}{c}\text { Falta de comunicación entre las } \\
\text { áreas }\end{array}$ & 1 & 0 & 0 & 0 & 0 & 2 & 3 & 6 \\
\hline 3 & $\begin{array}{l}\text { Falta de un macroproceso } \\
\text { documentado }\end{array}$ & 2 & 0 & 0 & 2 & 1 & 3 & 0 & 8 \\
\hline 4 & Poca confiabilidad del Software & 2 & 2 & 0 & 0 & 3 & 1 & 3 & 11 \\
\hline 5 & Exceso de documentación en físico & 2 & 0 & 1 & 3 & 0 & 1 & 3 & 10 \\
\hline 6 & $\begin{array}{l}\text { Quejas y reclamos de los usuarios, } \\
\text { familiares, y/o clientes }\end{array}$ & 0 & 1 & 0 & 2 & 2 & 0 & 3 & 8 \\
\hline 7 & Sobrecargo de responsabilidades & 3 & 3 & 2 & 0 & 2 & 2 & 0 & 12 \\
\hline \multicolumn{2}{|r|}{ Total Pasivo } & 10 & 9 & 5 & 8 & 11 & 11 & 15 & 69 \\
\hline
\end{tabular}

Fuente: Elaboración propia. 
1. Sobrecargo de responsabilidades.

2. Quejas y reclamos de los usuarios, familiares y/ o clientes.

\section{Exceso de documentación en físico}

A continuación, se realizó una clasificación de los siete factores estudiados, ubicándolos en las siguientes categorías: Problemas críticos, problemas pasivos, problemas indiferentes y problemas activos. Una vez clasificados se procedió a elaborar un diagrama que permitió observar la naturaleza de dichos problemas (Ver Fig. 3)

Como se puede observar el problema 4 (Poca confiabilidad del Software), es el único factor activo y es la causa raíz de los demás problemas, ya que al no haber un sistema confiable en el que se puedan generar los reportes y descargar la información, se está generando exceso de documentación en físico, y una sobrecarga laboral a los trabajadores del área, entre otros.
La desactualización delos documentos (Problema 1), también es un problema importantequetiene la institución, ya que está en el límite entre los factores críticos y los activos. Aquí se podría actualizar los procesos que se encuentran desajustados y documentar los que sean necesarios.

Dentro del problema 7 (Sobrecargo de responsabilidades), hay que tener presente la cantidad de tareas que se está dejando al personal de las diferentes áreas y hacer un reparto de actividades equitativo; todo ello se puede realizar analizando y reevaluando la función de los procesos de las diferentes áreas. También se observa, que esta sobrecarga es causada principalmente por la poca confiabilidad que se tiene en el Software que manejalainstitución.

Posteriormente, se aplicó al personal de la entidad, una encuesta estructurada, dónde el propósito era conocer la cantidad de conocimientos quelos empleados tenían sobre el Sistema de Gestión de Calidad delaentidad (SGC), Seguridad del Paciente

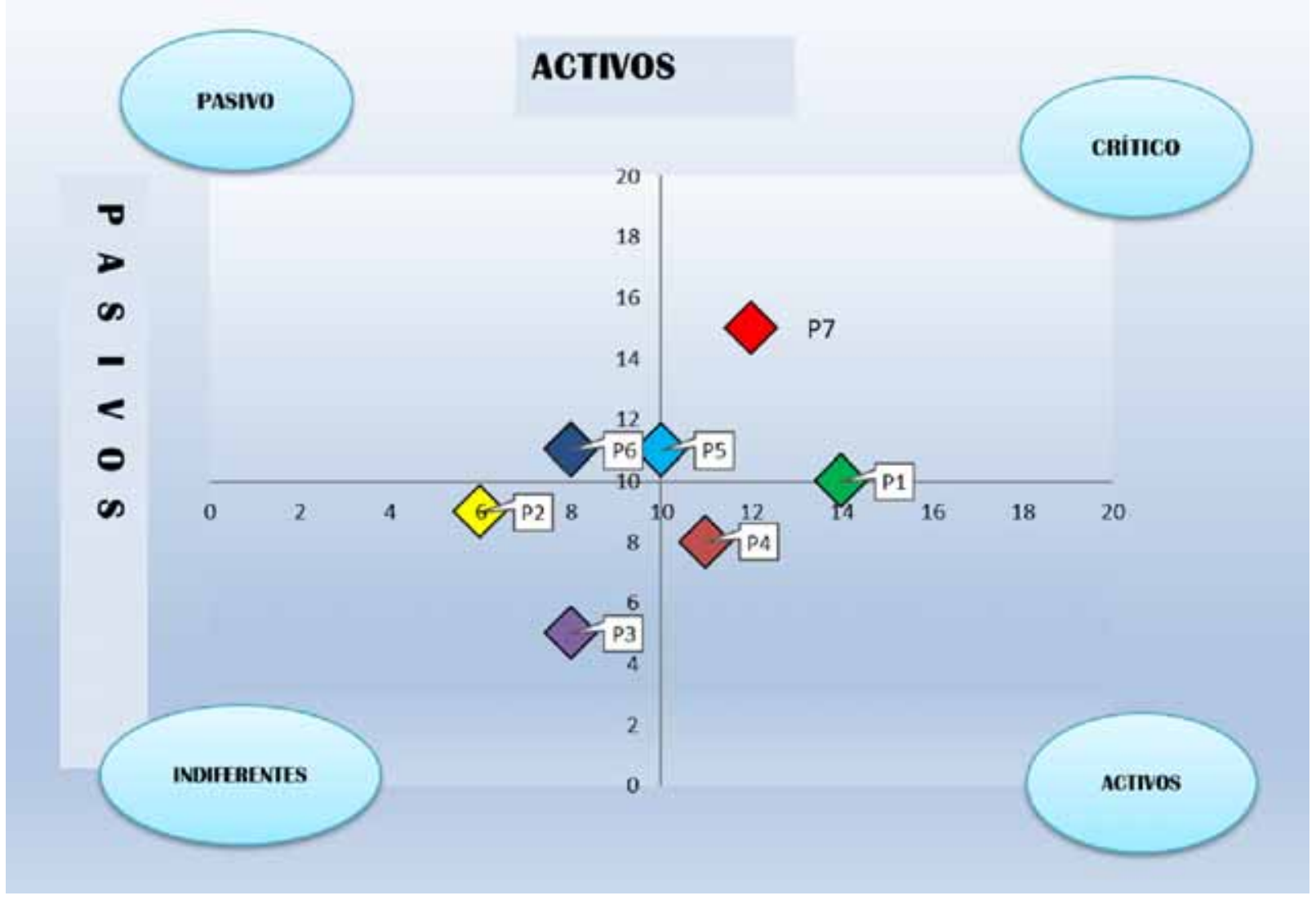

Fig. 3. Diagrama priorización de factores. Fuente: Elaboración propia. 
y SARLAFT. Para tal fin se realizaron una serie de preguntas, específicas en temas claves del área:

- ¿Sabeusted si existe un SGC?

- ¿Ha recibido capacitaciones sobreel SGC?

- ¿Conoce los temas de la política de calidad?

- ¿Reconocelos riesgos a los cuáles se encuentra expuesto el paciente?

- ¿Sabesusted cuál es el procedimiento para reportar un riesgo de Seguridad del Paciente?

- ¿ldentifica usted cuáles son los riesgos LA/ FT?

- ¿Conoce los controles del SARLAFT?

- ¿Conoce el procedimiento para reportar un riesgo LA/ FT?

Los resultados de dichas encuestas arrojaron que el personal de la entidad oncológica tiene conocimientos oportunos sobreel SGC, ya queen su total idad saben que hay un sistema de calidad y también en su mayoría conocen los temas de la política de calidad.

También se encontró que el $85 \%$ de los participantes, conocen la manera correcta de reportar un riesgo de Seguridad del Paciente oportunamente y el personal asistencial en un $100 \%$ reconoce los riesgos a los que se encuentran expuestos tanto ellos como los usuarios.

Además, se identificó que los empleados poseen conocimientos oportunos del SARLAFT, y que en un $75 \%$ deellos reconocen el procedimiento para reportar un riesgo de LA/ FT, en caso de que se presente.

\section{B. Documentación}

Tras realizar el diagnóstico se identificaron los flujos de información del proceso, los recursos, los actores que intervienen en las entradas, salidas, proveedores y clientes en cada uno de los proce dimientos con sus correspondientes responsables, todos estos elementos fueron necesarios para desarrollar la caracterización de los procesos.
Debido a la naturaleza de los procesos del área setomó la decisión de desarrollar dos caracterizaciones independientes, la primera para el SOGC (Sistema obligatorio de garantía de calidad) y la segunda para SARLAFT, ya que a pesar de que pertenezcan a una misma área y sus actividades son desarrollados por el mismo personal, tanto el objetivo como el alcance son direccionados a trabajar como procesos independientes.

Una vez identificadas las actividades dentro de las caracterizaciones, se establecieron los subprocesos pertenecientes a los procesos y se procedió a actualizar los procedimientos en conjunto con el área de Calidad, tomando como referente los formatos que se manejaban en ese momento en la institución, se realizaron ajustes y modificaciones en cuanto a forma de los documentos, contenido mínimo deacuerdo a su tipo, codificación por procesos de acuerdo al organigrama dela institución, las directrices a tener en cuenta para la elaboración, entre otros, lo anterior con el fin de cumplir el objetivo de lograr la estandarización a nivel institucional.

El primer paso fue realizar la revisión de los documentos que se manejaban en ese momento y compararlos con las actividades identificadas en el momento de establecer la caracterización, por lo que se validó a través de la relación que debían tener, (Ver Fig. 4), donde cada documento de apoyo (Formatos, anexos, guías, manuales, entreotros) se debía encontrar relacionado a un procedimiento y este a su vez debía pertenecer a al guno de los subprocesos del área.

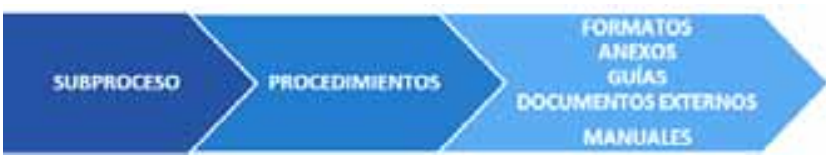

Fig. 4. Relación documental Fuente: Elaboración propia.

Después de realizar el barrido de información se observó que existían documentos de apoyo que según su vigencia se encontraban activos, pero no se utilizaban; registros que eran usados, pero no estaban codificados ni relacionados en los listados maestros; procedimientos que no se realizaban de acuerdo a lo que estaba descrito, sino que el profesional los ejecutaba de otra manera valiéndose de otras herramientas o pasos que no se encontraban documentados. 
Debido a los elementos mencionados anteriormente fue necesario establecer 3 categorías; “Activos" para los documentos vigentes, estos podían ser tanto los documentos que ya se encontraban en la institución y sedebían validar y actualizar, como los documentos que se debían desarrollar desde cero, "Cambio de área" para aquellos que tenían queser ubicados dentro delos procesos deotraárea por su contenido o ejecución y "Eliminado" queson aquellos que pertenecían al área, pero ya no hacían parte de la operación y debían ser dados de baja, como serepresenta en el siguiente gráfico (Ver Fig. 5), donde los documentos el iminados o cambiados de área representan el $14 \%$ de ellos, mientras que 174 documentos permanecen activos.

A continuación, se observa una gráfica donde se evidencia la comparación de la cantidad de documentos en el año 2018 y el año 2020, es decir el antes y después del proceso de estandarización (Ver Fig. 6).

En cuanto a documentos activos, se observa un considerable aumento después de realizar el proceso de estandarización de aproximadamente un $46 \%$ con respecto al año 2018 con unos picos resal tables en instructivos y documentos externos, estos últimos se debe a que se relacionaban en los diferentes procedimientos, pero no se encontraban codificados ni relacionados en el listado maestro de documentos.
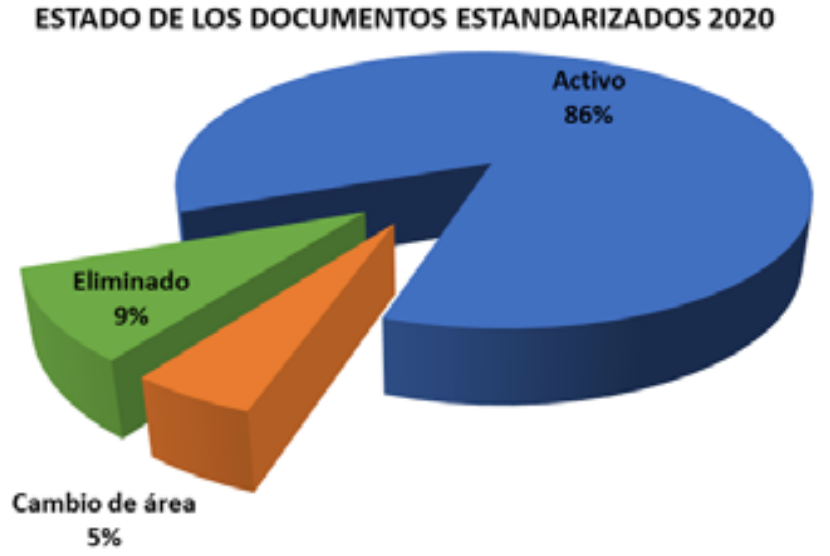

Fig. 5. Clasificación de los documentos. Fuente: Elaboración propia.

También se observa el aumento en el número deformatos quees proporcional al crecimiento de los procedimientos, ya que de las 28 operaciones que se encuentran actualmente el $32 \%$ no estaban estandarizadas, sino que se ejecutaban sin estar documentadas.

Una vez estandarizados los documentos, se identificaron los riesgos a los cuales el área degestión de Calidad, Seguridad del Paciente y SARLAFT se encontraban expuestos, por lo que en colaboración con la institución se aplicó una matriz de riesgos basada en la norma ISO 31000 [16], donde se relacionaron los riesgos vinculados a cada uno de los procedimientos.

\section{DOCUMENTACIÓN ÁREA 2018 vs 2020}

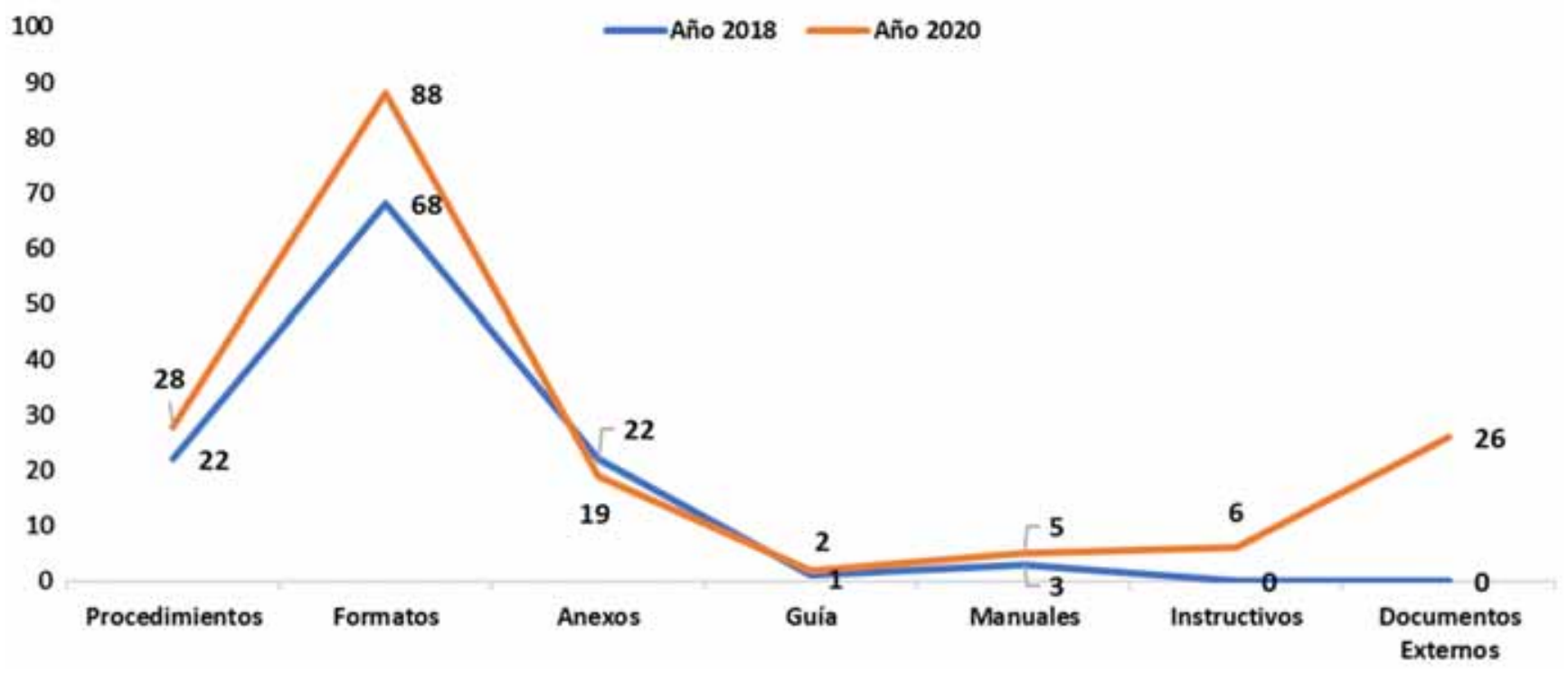

Fig. 6. Comparación documentos del área antes y después del proceso de estandarización. Fuente: Elaboración propia. 
Cada uno de los riesgos propuestos fueron relacionados con las causas y efectos correspondientes, además, de las calificaciones de impacto y probabilidad para determinar la severidad y el riesgo inherente.

Para el cálculo del riesgo residual en colaboración con el área se establecieron los controles correspondientes al riesgo, el tipo, la naturaleza y la frecuencia del control.

Como resultado, se calcularon en total 54 riesgos para los 28 procedimientos, los cuales se pueden observar la Fig. 7.

\section{Tiempos y estandarización}

Para realizar el estudio de tiempos dentro del área lo primero que se realizó fue la clasificación de actividades en rutinarias, donde se identificaron 4 procedimientos con estas características, y no rutinarias que corresponde al $86 \%$ del total de operaciones que se desarrollan en el área como se evidencia en el Fig. 8.

Para el cálculo de las actividades rutinarias se realizó un estudio detiempos basado en tomas con cronómetro, en observaciones de acuerdo a la metodología propuesta por Criollo [11].
El número de observaciones variaba de acuerdo a las 10 mediciones realizadas inicial mente, para el caso de las actividades evaluadas los rangos se encontraban entre 9 y 17 tomas, para lo cual se diseñó un formato donde se consolidó la información de las tomas, además de datos como la valoración dedesempeño y el valor delos suplementos establecidos.

Para las actividades no rutinarias se consultaron 3 expertos quecontarán con experiencia en los procesos del área y preferiblemente que pertenecieran al sector salud, con el objetivo de que las actividades a evaluar puedan ser afines a las operaciones desarrolladas por la institución, debido a las características del os procesos queabarca el área se estableció la división en 3 grupos, como lo son: Gestión de calidad, seguridad del paciente y SARLAFT, y cada uno de estos sería consultado con las características mencionadas anteriormente, estos expertos serán nombrados como “Expertos externos".

Adicional a esto, se tomó el tiempo interno en el desarrollo de las actividades y fueron establecidos como "Expertos internos".

Se evaluaron los tiempos de los expertos y se generó un tiempo estándar, donde se observa que

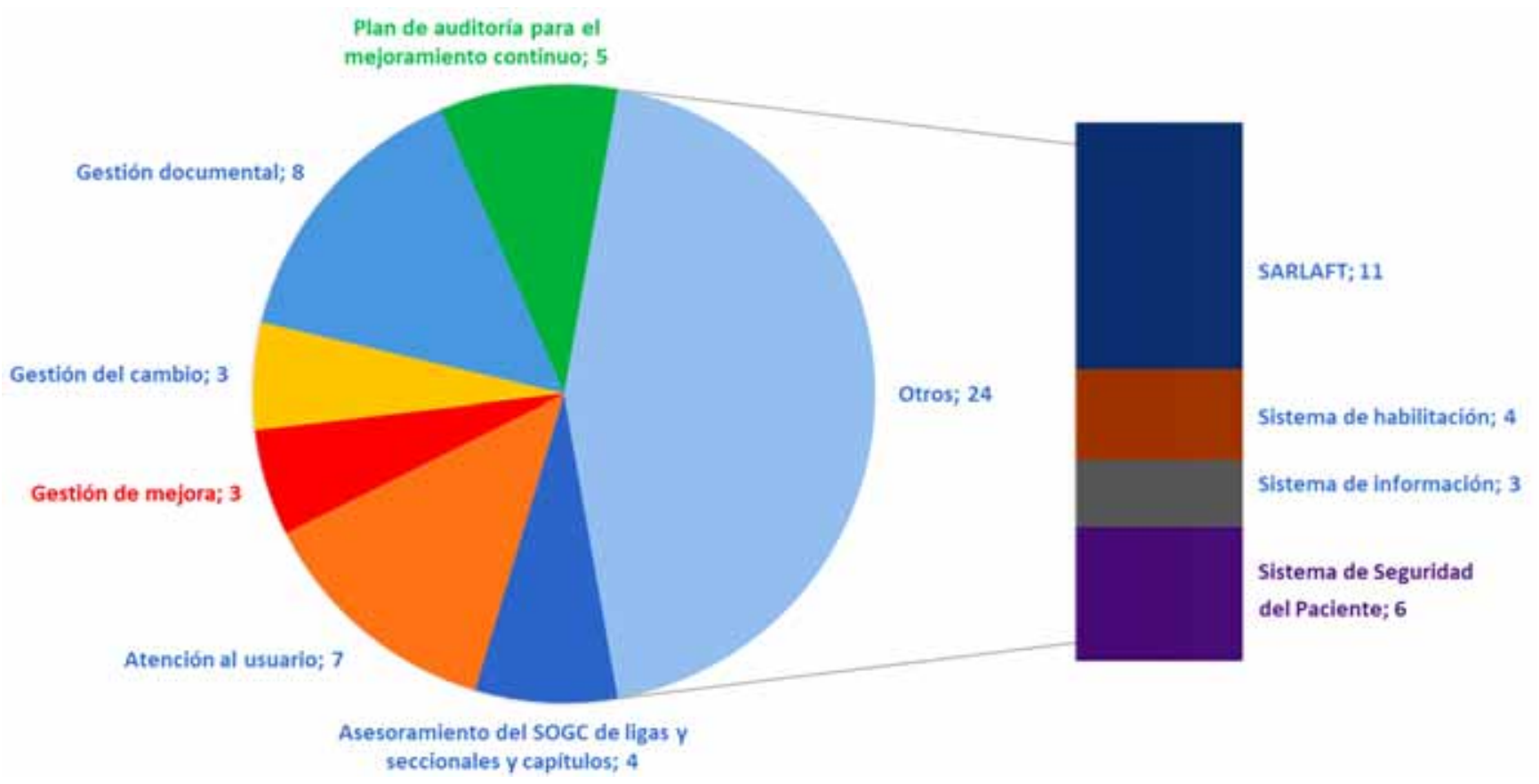

Fig. 7. Distribución de riesgos de acuerdo al subproceso. Fuente: Elaboración propia. 


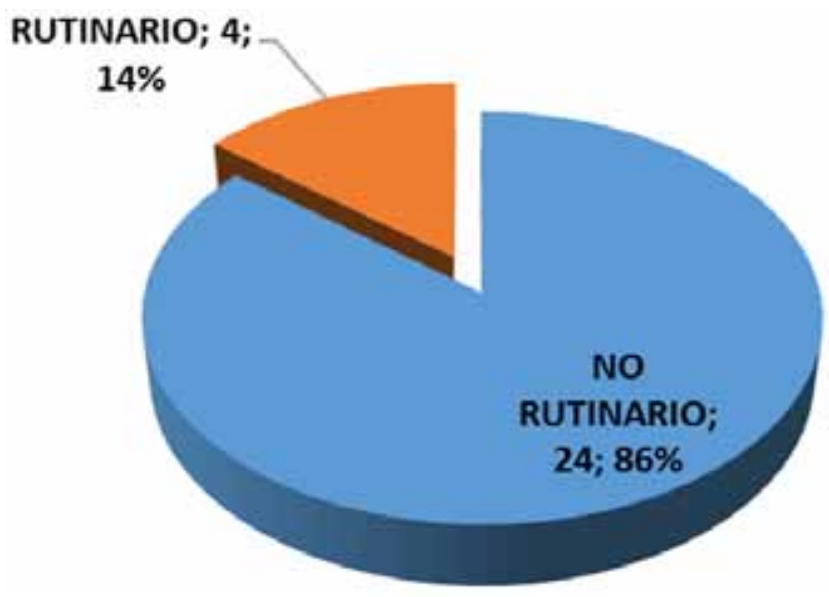

Fig. 8. Clasificación de tiempos del área. Fuente: Elaboración propia

el 56\% de las actividades del área seencuentra por encima del parámetro; es decir, 14 de los procedimientos real izados a nivel interno son superiores al patrón.

El $44 \%$ restante equivale a las actividades dondela institución seencuentra por debajo del tiempo estándar lo que refleja la efectividad de casi la mitad desus actividades frenteal patrón, pero con la misma observación que los procedimientos anteriores, un aumento considerable de los tiempos en los pasos de seguimiento y control.

Como resultado del análisis realizado anteriormente, seobtuvo el tiempo estándar final y seagrupó por los subprocesos dentro del área, donde se evidencia el comportamiento de los tiempos obtenidos tras el estudio (Ver Fig. 9)

\section{Indicadores}

Una vez estandarizados los procesos, el área debía asegurar el control efectivo de los procedimientos, así como los métodos necesarios para garantizar el funcionamiento óptimo de las actividades con un enfoque en la mejora continua, para esto fue necesario desarrollar una herramienta basada en indicadores claves de rendimiento.

Lainstitución tenía implementada una metodología de seguimiento para indicadores de gestión, la cual consistía, en el diligenciamiento de la hoja de vida del indicador de acuerdo a la periodicidad estipulada y semestralmente se presentaban resultados a través de un informe de gestión o una presentación ejecutiva.

Al realizar la validación de la hoja del indicador se observó que no era del todo clara ni defácil manejo, por lo que se detectó la necesidad de desarrollar una herramienta que facilitara el diligenciamiento, el control, seguimiento y análisis de los indicadores.

Una vez estandarizado el formato y ficha técnica de indicadores se procedió a adaptar los indicadores del área de Calidad, Seguridad del Paciente y SARLAFT. Como resultado se ajustaron 22 indicadores clasificados en indicadores de productividad (Ver Fig. 10).

Losindicadores establecidos permiten queel área lleveun control del desempeño delos procesosevaIuados dentro de la institución y de igual manera facilite la trazabilidad, lo que permita generar

\section{TIEMPOS ESTANDAR VS TIEMPOS INSTITUCIÓN}

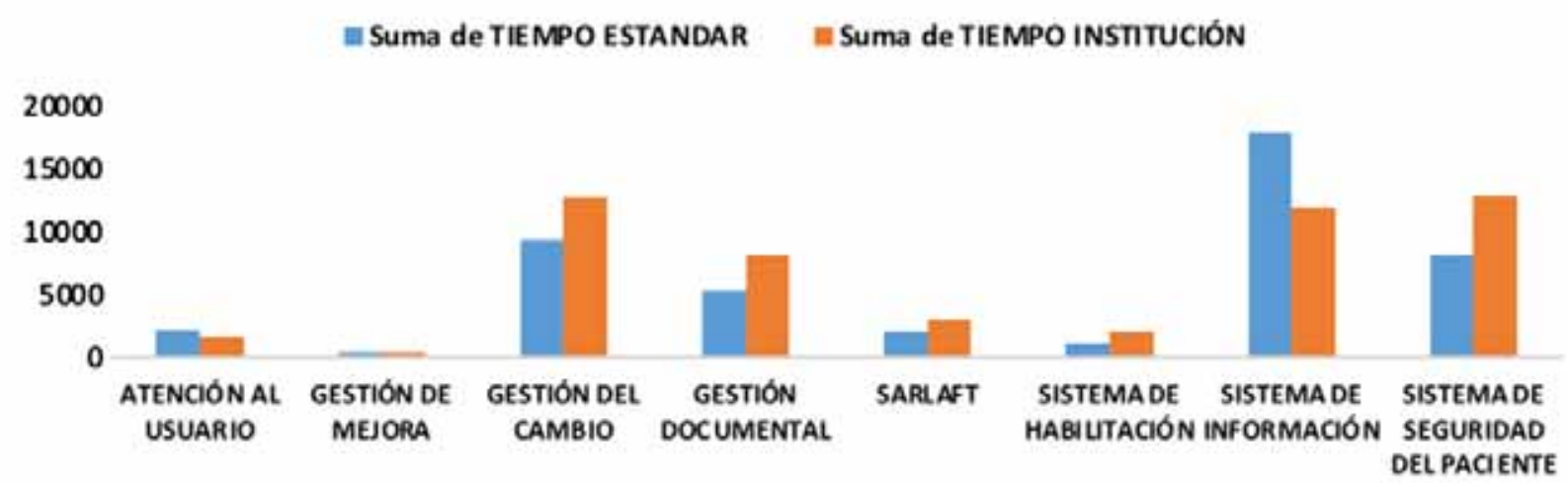

Fig. 9. Distribución de riesgos de acuerdo al subproceso. Fuente: Elaboración propia. 


\section{CLASIFICACIÓN INDICADORES ÁREA DE CALIDAD,SEGURIDAD DEL PACIENTE Y SARLAFT}

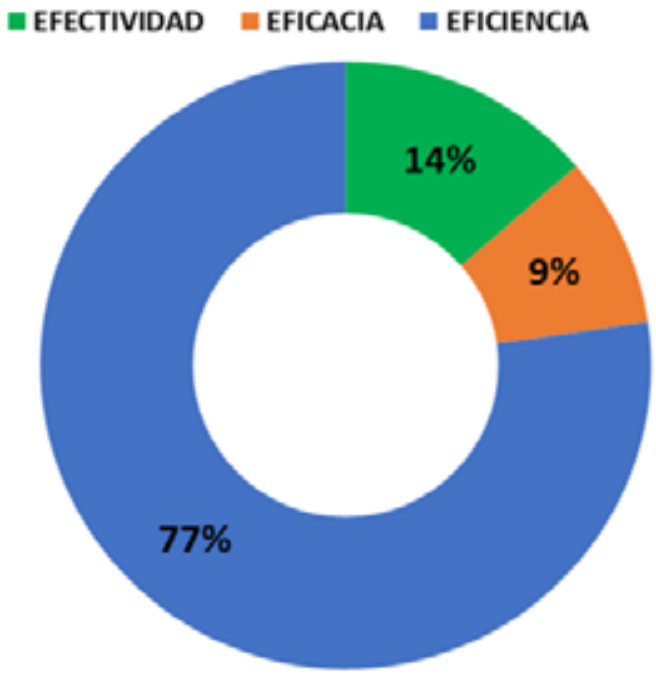

Fig. 10. Clasificación de indicadores de acuerdo al tipo de actividad. Fuente: Elaboración propia. estrategias ligadas al cumplimiento y ejecución de Ios objetivos, así como la facilidad al identificar los puntos críticos quegeneran las desviaciones en cuanto a la meta, permitiendo el planteamiento de acciones de seguimiento y de mejora.

\section{E. A nálisis de costos}

Por último, era necesario evaluar el impacto que tuvo el desarrollo del proyecto tanto en el área como en la institución, por lo queteniendo en cuenta las operaciones del área se decidió aplicar un análisis de costos de calidad a través de la implementación del modelo PEF desarrollado por Feigenbaum [17].

Teniendo en cuenta la definición de las categorías necesarias para calcular el costo total de calidad, el primer paso fue identificarlas en el área de estudio (Ver tabla II).

Tabla II. Categorías del costo en el área.

\begin{tabular}{|c|c|c|c|c|c|c|c|}
\hline Elemento & Prevención & Elemento & Evaluación & Elemento & $\begin{array}{l}\text { Fallos } \\
\text { internos }\end{array}$ & Elemento & $\begin{array}{c}\text { Fallas } \\
\text { externas }\end{array}$ \\
\hline P1 & $\begin{array}{l}\text { Comité de } \\
\text { Calidad }\end{array}$ & E1 & $\begin{array}{l}\text { Auditorías } \\
\text { de calidad }\end{array}$ & FI1 & Reprocesos & FE1 & $\begin{array}{l}\text { Gestión de } \\
\text { quejas y } \\
\text { reclamos }\end{array}$ \\
\hline $\mathrm{P} 2$ & $\begin{array}{c}\text { Auditoría } \\
\text { interna }\end{array}$ & E2 & $\begin{array}{c}\text { Auditorías } \\
\text { externas }\end{array}$ & FI2 & $\begin{array}{c}\text { Tiempo } \\
\text { extra }\end{array}$ & FE2 & $\begin{array}{l}\text { Gestión de } \\
\text { tutelas }\end{array}$ \\
\hline P3 & Mejora continua & E3 & $\begin{array}{l}\text { Rondas de } \\
\text { seguridad }\end{array}$ & FI3 & $\begin{array}{l}\text { Tiempos } \\
\text { de ocio }\end{array}$ & FE3 & $\begin{array}{c}\text { Gestión de } \\
\text { requerimien } \\
\text { tos de entes } \\
\text { de control } \\
\text { preventivo }\end{array}$ \\
\hline P4 & $\begin{array}{l}\text { Inducción y } \\
\text { capacitación }\end{array}$ & $\mathrm{E} 4$ & $\begin{array}{c}\text { Reporte de } \\
\text { eventos }\end{array}$ & FI4 & $\begin{array}{l}\text { Eventos } \\
\text { adversos }\end{array}$ & FE4 & $\begin{array}{c}\text { Gestión de } \\
\text { requerimien } \\
\text { tos de entes } \\
\text { de control } \\
\text { correctivo }\end{array}$ \\
\hline P5 & $\begin{array}{l}\text { Estandarización } \\
\text { de documentos }\end{array}$ & E5 & $\begin{array}{c}\text { Medición } \\
\text { de } \\
\text { adherencia } \\
\text { de } \\
\text { procesos }\end{array}$ & FI5 & \multicolumn{2}{|c|}{$\begin{array}{l}\text { Incumplimiento de } \\
\text { procesos }\end{array}$} & \\
\hline P6 & $\begin{array}{c}\text { Planificación de } \\
\text { Calidad }\end{array}$ & E6 & $\begin{array}{c}\text { Validación } \\
\text { de } \\
\text { procesos }\end{array}$ & & & & \\
\hline P7 & $\begin{array}{c}\text { Comité de } \\
\text { Seguridad del } \\
\text { Paciente }\end{array}$ & E7 & $\begin{array}{l}\text { Búsqueda } \\
\text { activa de } \\
\text { casos }\end{array}$ & & & & \\
\hline
\end{tabular}

Fuente: Elaboración propia. 
Una vez identificadas las categorías se relacionaron los costos queintervenían en su desarrollo, para lo cual fuenecesario conocer el costo por hora delos diferentes actores que participan en los elementos, adicional se estableció el valor del costo de insumos que debido a la naturaleza delas actividades seestableció como un costo fijo, un costo de servicio de carácter variabley un último valor por concepto de otros gastos, esteúltimo se decidió debido a la necesidad específica dealgunos elementos.

Para identificar los actores se recurrió a metodologías deinvestigación científica principalmentela herramienta cual itativa como es la observación y para algunos elementos se aplicó la indagación directa.

De acuerdo a autores como Hernández [18] la investigación tienecomo objetivo no solo comprender procesos y la vinculación de las personas en el desarrollo de las circunstancias, sino identificar problemas y explorar los contextos presentes en las situaciones evaluadas, para lo que seaplicó las estrategias queel sugiere para quién realiceobservación sin ser experto.

A ntes de aplicar alguna de las herramientas mencionadas anteriormente, seclasificaron los ele mentos de las categorías a manera de separar los que eran necesario evaluar a través de observación y los que requerían indagación. Para este segundo escenario, se estructuró una entrevista basada en un caso y seconsultó a los profesionales encargados del proceso.

Como resultado de la investigación se obtuvo lo siguiente:

- La participación de 13 actores como se evidencia en la siguiente tabla (Ver tabla III).

- El promedio en horas que interviene cada uno de los participantes de acuerdo al elemento evaluado.

- Para el cálculo dehoras persona involucrada setomó en cuenta variables de tiempo como suplementos con el mismo peso porcentual que el estudio de tiempos.

Después de identificar tanto actores, como ele mentos de categorías, se consolidó la información
Tabla III. Codificación actores involucrados.

\begin{tabular}{|l|c|}
\hline \multicolumn{1}{|c|}{ CARGO } & CÓDIGO \\
\hline $\begin{array}{l}\text { Director adscrito para la } \\
\text { Calidad }\end{array}$ & DC \\
\hline Profesional de Calidad & PC \\
\hline $\begin{array}{l}\text { Auxiliar de gestión } \\
\text { documental }\end{array}$ & AC \\
\hline Director médico & DM \\
\hline Enfermera oncóloga & EO \\
\hline Médico oncólogo & MO \\
\hline Químico farmacéutico & QF \\
\hline Presidente & PG \\
\hline $\begin{array}{l}\text { Profesional involucrado } \\
\text { directo }\end{array}$ & PI \\
\hline Otro profesional & OP \\
\hline Costos de insumos & CI \\
\hline Costo de servicio & CS \\
\hline Otro gasto & OG \\
\hline
\end{tabular}

Fuente: Elaboración propia.

obtenida en la investigación donde seevidencia la relación entre actores y elementos para cal cular el costo unitario por elemento, como se muestra a continuación (Ver tabla IV).

Para el cál culo de cada uno de los elementos se aplicó la siguientefórmula:

$$
\sum_{i=1}^{n}(\operatorname{Vh} x(t+(t * 15 \%)))+C I+C S+O G
$$

Fig. 11. Fórmula frecuencia [15]

Donde:

Vh: Valor hora por actor

t: Tiempo en horas

CI: Costos de insumos

CS: Costos de servicio

OG : Otros gastos

Después de cal cular los costos de cada uno de los elementos se procedió a implementar la metodología PEF en los procesos estandarizados del área de Calidad, Seguridad del Paciente y SARLAFT, donde se tomó como insumo la matriz de riesgos desarrollada anteriormente, priorizando los subprocesos del área de acuerdo a su severidad. 
Tabla IV. Matriz de elementos vs actores.

\begin{tabular}{|c|c|c|c|c|c|c|c|c|c|c|c|c|c|c|}
\hline \multirow{2}{*}{\multicolumn{2}{|c|}{ Elementos vs actores }} & \multicolumn{10}{|c|}{ Tarifa horaria } & \multirow{3}{*}{ CI } & \multirow{3}{*}{ CS } & \multirow{2}{*}{ OG } \\
\hline & & & & $\Delta$ & DM & & & OF & & & PQ & & & \\
\hline Elemento & Prevención & & & & & & & & & & & & & \\
\hline P1 & Comité de Calidad & $X$ & $X$ & & $X$ & & & & $X$ & & $X$ & $X$ & & \\
\hline P2 & Auditoría interna & $X$ & & & & & & & & $X$ & $X$ & & & \\
\hline P3 & Mejora continua & $X$ & & & & & & & & $X$ & $X$ & $X$ & & \\
\hline $\mathrm{P} 4$ & Inducción y capacitación & $X$ & $\mathrm{X}$ & & & & & & & & & $X$ & & \\
\hline P5 & Estandarización de documentos & $X$ & $X$ & $X$ & & & & & & $X$ & & $X$ & & \\
\hline P6 & Planificación de Calidad & $X$ & $X$ & & & & & & $X$ & & & $X$ & & \\
\hline P7 & Comité de Seguridad & $X$ & & & $X$ & $X$ & $X$ & $X$ & & & & $X$ & & \\
\hline Elemento & Evaluación & & & & & & & & & & & & & \\
\hline E1 & Auditorías de calidad & $\mathrm{X}$ & & & & & & & & & $\mathrm{X}$ & & & \\
\hline E2 & Auditorías externas & $X$ & & & & & & & & & $X$ & & & \\
\hline E3 & Rondas de seguridad & $X$ & & & & $X$ & $X$ & $X$ & & & $X$ & $\mathrm{X}$ & & \\
\hline E4 & Reporte de eventos & $X$ & & & $X$ & $X$ & $X$ & $X$ & & & $X$ & $X$ & & \\
\hline E5 & Medición de adherencia de procesos & $X$ & & & & $X$ & $X$ & $X$ & & & $X$ & & $\mathrm{X}$ & \\
\hline E6 & Validación de procesos & $X$ & $X$ & & & & & & & $X$ & & $X$ & & \\
\hline E7 & Búsqueda activa de casos & $X$ & $X$ & & & $X$ & $X$ & $X$ & & & $\mathrm{X}$ & $X$ & & \\
\hline Elemento & Fallos internos & & & & & & & & & & & & & \\
\hline FI1 & Reprocesos & & & & & & & & & $X$ & & $X$ & & \\
\hline FI2 & Tiempo extra & $X$ & $\mathrm{X}$ & & & & & & & $X$ & & & & \\
\hline FI3 & Tiempos de ocio & & & & & & & & & & $X$ & $X$ & & \\
\hline FI4 & Eventos adversos & $X$ & & & $X$ & $X$ & $X$ & $X$ & & & $X$ & $X$ & $X$ & \\
\hline FI5 & Incumplimiento del proceso & $X$ & & & & & & & & $X$ & & & & \\
\hline Elemento & Fallos externos & & & & & & & & & & & & & \\
\hline FE1 & Gestión de quejas y reclamos & $\mathrm{X}$ & $\mathrm{X}$ & & & & & & $X$ & $X$ & & $\mathrm{X}$ & & $X$ \\
\hline FE2 & Gestión de tutelas & $X$ & $X$ & & & & & & $X$ & $X$ & & $X$ & & $X$ \\
\hline FE3 & $\begin{array}{l}\text { Gestión de requerimientos de entes } \\
\text { de control preventivo }\end{array}$ & $\mathrm{X}$ & $X$ & & & & & & $x$ & $\mathrm{X}$ & & $X$ & & $X$ \\
\hline FE4 & $\begin{array}{l}\text { Gestión de requerimientos de entes } \\
\text { de control correctivo }\end{array}$ & $x$ & $X$ & & & & & & $X$ & $X$ & & $X$ & & $X$ \\
\hline
\end{tabular}

Fuente: Elaboración propia basado en Tapia \& Rodolfo [15]

Priorizado los subprocesos se observa que aproximadamente el $62 \%$ de los riesgos estaban presentes en cuatro (4) subprocesos, estos son: Gestión documental (19\%), atención al usuario (17\%), sistema de seguridad del paciente (15\%) y sistema de habilitación con (11\%), (Ver Fig. 12).

Una vez priorizados los subprocesos, aplicando una matriz se relacionaron los riesgos, con los elementos para cal cular el costo total de calidad, a continuación, se observa un ejemplo de la aplicación de la matriz para algunos de los riesgos relacionadosal subproceso degestión documental (Ver tabla V). Debido a la sensibilidad de los datos re colectados se presentan en valor porcentual con respecto al total del costo de calidad.
Después de determinar los costos de calidad según el subproceso y sus riesgos se identificaron 3 riesgos que al observar sus costos debido a su comportamiento diferentese analizaron demanera independiente a los costos totales de calidad (Ver tabla VI).

La desviación de costos al aplicar la metodología es debido a los costos de fallos externos en caso de incurrir en al guno de estos tres riesgos, ya que pueden ser sanciones económicas de hasta 10.000 SMMLV, lo cual abarcaría un gran riesgo para la entidad.

Por otro lado, teniendo en cuentas las excepciones mencionadas anteriormente el resultado de 


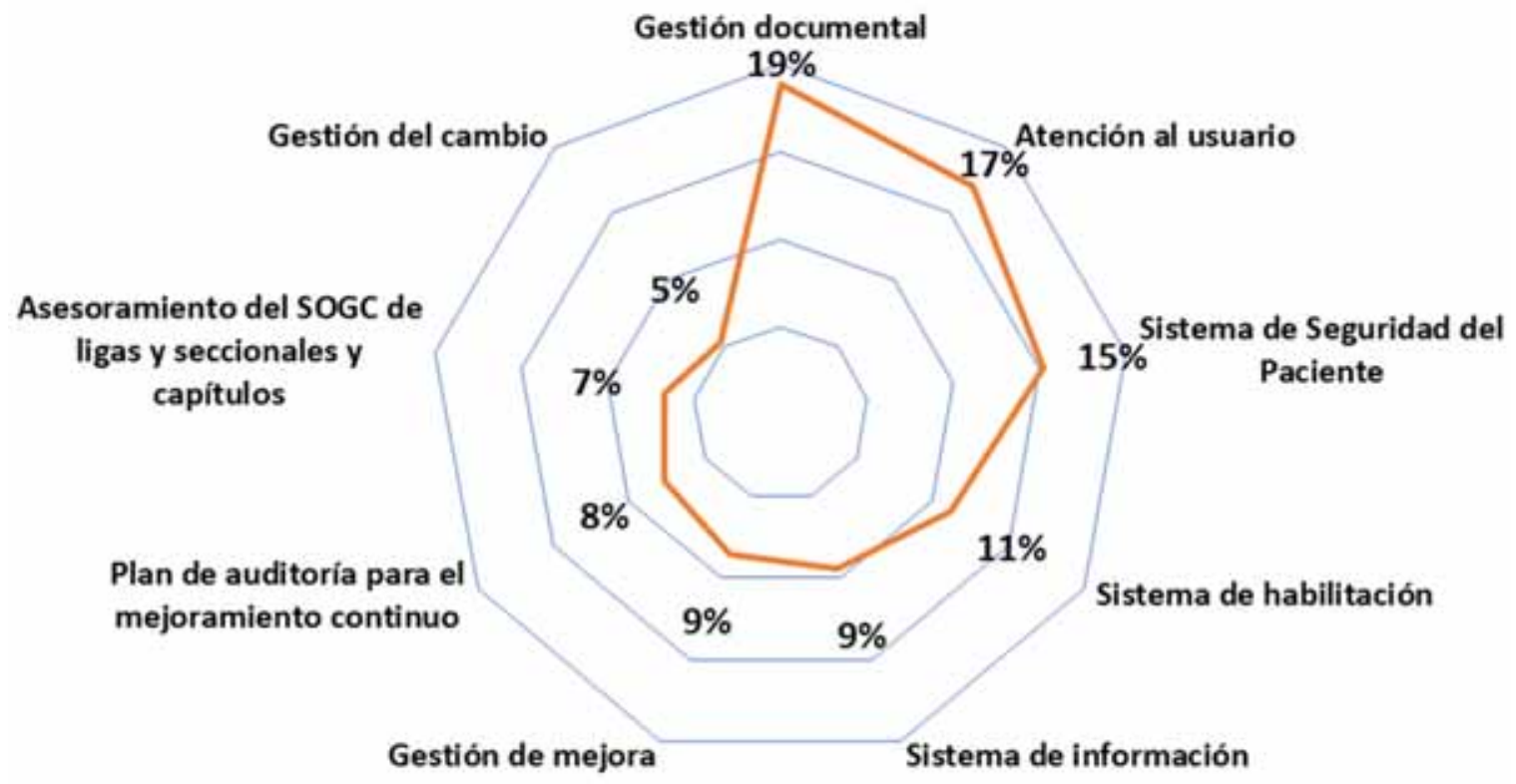

Fig. 12. Diagrama de priorización de riesgos. Fuente: Elaboración propia

Tabla. V. Matriz de riesgos y costos de calidad.

\begin{tabular}{|c|c|c|c|c|c|c|c|c|c|c|}
\hline 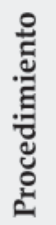 & 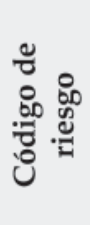 & 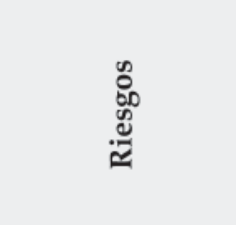 & 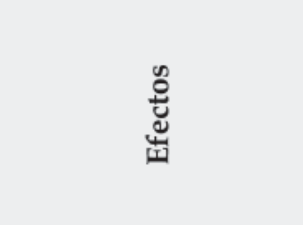 & कृ & 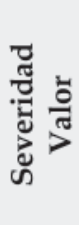 & 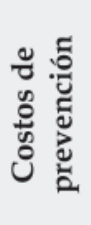 & 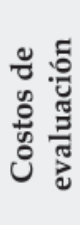 & 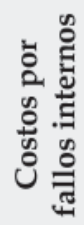 & 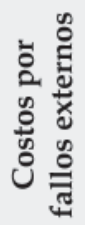 & 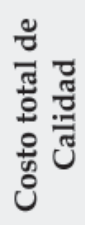 \\
\hline \multirow[b]{2}{*}{$\begin{array}{l}\bar{\delta} \\
u \\
0 \\
0 \\
0 \\
0\end{array}$} & 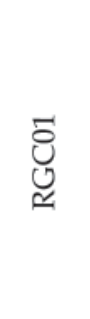 & $\begin{array}{l}\text { Desvío de } \\
\text { parámetros y } \\
\text { lineamientos en } \\
\text { la forma de los } \\
\text { documentos } \\
\text { establecidos } \\
\text { por la } \\
\text { Institución }\end{array}$ & $\begin{array}{l}\text { Incumplimiento del } \\
\text { procedimiento }\end{array}$ & Moderado & 2,5 & $51 \%$ & $3 \%$ & $46 \%$ & $0 \%$ & $100 \%$ \\
\hline & 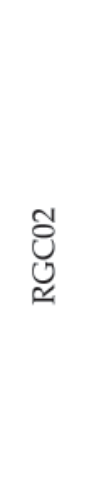 & $\begin{array}{l}\text { Creación de } \\
\text { documentos } \\
\text { (Procedimiento, } \\
\text { manuales, } \\
\text { formatos, } \\
\text { anexos, entre } \\
\text { otros) que no se } \\
\text { encuentren } \\
\text { avalados por el } \\
\text { área de Calidad } \\
\text { y estén siendo } \\
\text { usados por el } \\
\text { personal }\end{array}$ & $\begin{array}{l}\text { Incumplimiento del } \\
\text { procedimiento } \\
\text { Ausencia de } \\
\text { seguimiento debido } \\
\text { a que no se } \\
\text { encuentra } \\
\text { estandarizado y } \\
\text { documentando en el } \\
\text { listado maestro de } \\
\text { documentos }\end{array}$ & Alto & 3 & $63 \%$ & $9 \%$ & $14 \%$ & $14 \%$ & $100 \%$ \\
\hline
\end{tabular}

Fuente: Elaboración propia.

Rev. Ingeniería, M atemáticas y Ciencias de la Información Vol. 7 / Núm. 14 / julio - diciembre de 2020; pág. 39-57 
Tabla V. Riesgos con comportamiento diferente

\begin{tabular}{|c|c|c|c|c|c|c|}
\hline Procedimiento & $\begin{array}{l}\text { Código de } \\
\text { riesgo }\end{array}$ & Riesgos & Efectos & Severidad & $\begin{array}{c}\text { Severidad } \\
\text { Valor }\end{array}$ & $\begin{array}{l}\text { Costo total de } \\
\text { Calidad }\end{array}$ \\
\hline PRO GC 010 & RGC14 & $X$ & $\begin{array}{l}\text { Repercusiones legales } \\
\text { y reputacionales }\end{array}$ & Alto & 3 & $\begin{array}{l}\text { Sumas más de } \\
600 \text { SMMLV }\end{array}$ \\
\hline \multirow{2}{*}{ PRO GC 017} & RGC09 & $X$ & $\begin{array}{l}\text {-Inactivación de la } \\
\text { inscripción } \\
\text {-Visita de reactivación } \\
\text {-Sanciones legales }\end{array}$ & Alto & 3 & $\begin{array}{l}\text { Sumas más de } \\
600 \text { SMMLV }\end{array}$ \\
\hline & RGC10 & $X$ & $\begin{array}{lll}\text {-Cierre } & \text { de } & \text { la } \\
\text { institución } & & \\
\text {-Sanciones } & & \\
\text { económicas } & & \end{array}$ & Extremo & 4 & $\begin{array}{l}\text { Sumas más de } \\
600 \text { SMMLV }\end{array}$ \\
\hline
\end{tabular}

Fuente: Elaboración propia.

los costos de no calidad (costos por fallos internos y externos), señala que en caso de presentarse estos riesgos los costos de no calidad representan el 30\% del costo total (Ver Fig.13).

A continuación, (Ver Fig.14) se observa el comportamiento de los costos de acuerdo a las categorías. Como tendencia se identifica la importante participación delos costos de prevención en general de los riesgos detectados, también se evidencia quemientras el costo deevaluación disminuye los costos por fallos internos aumentan considerablemente, lo queimplica que una delas estrategias para gestionar estos riesgos es realizar inversión en los elementos de valoración como herramientas para mitigar estos riesgos.

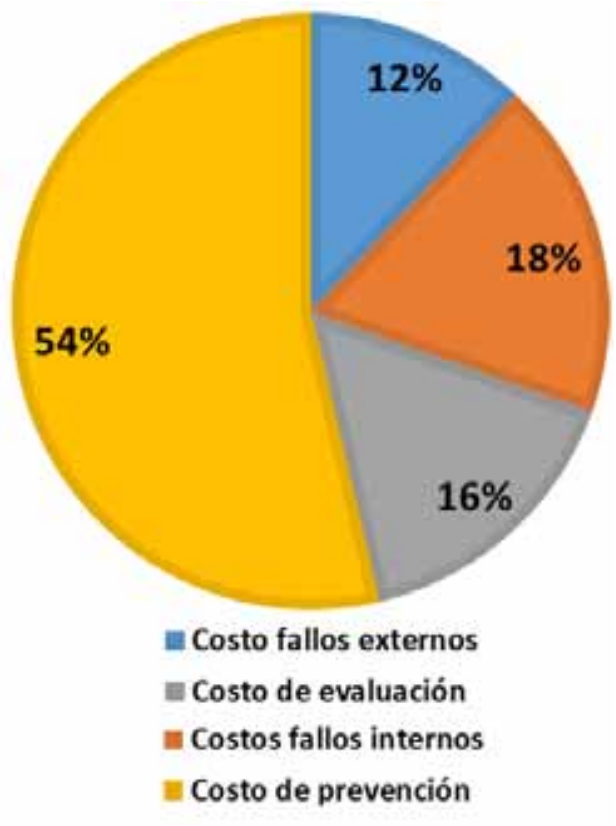

Fig.13. Distribución costo total de calidad. Fuente: Elaboración propia.
RGC33

RGC31

$\operatorname{RGC30}$

RGC28

RGC15

RGC01

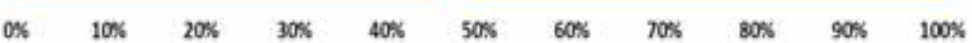

= Suma de Costo fallos externos total

= Suma de Costo evaluación total

= Suma de Costo fallos internos total

in Suma de Costo prevención total

Fig. 14. Distribución del costo total de calidad. Fuente: Elaboración propia.

Rev. Ingeniería, M atemáticas y Ciencias de la Información Vol. 7 / Núm. 14 / julio - diciembre de 2020; pág. 39-57 


\section{Discusión}

Uno de los problemas principales que se tuvo para hacer esta investigación, fue la ausencia de referencias respecto al tema de estandarización de procesos en el sector salud y puntualmente en las áreas de Calidad, Seguridad del Paciente y SARLAFT. Para solucionar dicha barrera, se optó por adaptar metodologías y herramientas planteadas por algunos autores aplicadas en otros sectores y contextos diferentes (Como el sector textil).

Tomando como referencia, el estudio realizado por Pérez [5] dondeal evidenciar inconformidades en el desarrollo de los procesos y la falta de documentación y estandarización en general delas actividades, el autor desarrolló, una metodología basada en 7 pasos orientada al control de escritos a través de listados maestros.

En esteproyecto el autor documentó inicial mentelos procedimientos identificados, aplicando herramientas de investigación, principalmente observación y medición de tiempos de las operaciones, finalizando con la caracterización del proceso y los controles pertinentes.

A pesar de que esta metodología cuenta con una serie de pasos organizados estos son orientados a la estandarización de procesos en un ambiente con poca variabilidad y con actividades repetitivas, donde el patrón de desarrollo sigue una tendencia definida en el proceso de producción textil, además de que se plantea como una metodología para el proceso de documentación desde cero, es decir que no se cuenta con registros o una base del proceso.

Debido a las características del área y del sector en el que se esta desarrollando el presenteproyecto surgió la necesidad de adaptar algunas de las herramientas propuestas por Pérez [5], pero direccionándolo a la condición actual del área donde secontaba con una base documental queservía deguía, por lo queel pilar principal del desarrollo del trabajo fue la caracterización de los procesos y seguido a esto se pudo documentar adecuadamente cada actividad.

En la parte detoma de tiempos, una vez consolidada la información obtenida en cada uno de los subprocesos, y ajustadas las variables del peso porcentual de acuerdo a las características del experto, se identificó que de los 28 procedimientos que realiza el área, 3 procedimientos no pudieron ser evaluados a través de "la metodología de cargas de trabajo" Universidad N acional [12].

Dos de estos procedimientos no se tuvieron en cuenta debido a las competencias y experticia que se requieren para realizar este tipo de actividades, estos son relacionados con asesoramiento de sistemas e implementación de proyectos. La otra actividad queno pudo ser evaluada fuea causa dela desviación de los datos entre los tiempos de los expertos externos, ya que no generaban confianza en el momento de ser consolidados.

De igual manera, un punto de divergencia entre este trabajo y la investigación realizada por Pérez [5], es la evaluación de valor que tuvo la estandarización del proyecto en el área y a nivel institucional . Autores como Tapia \& Rodolfo [15] aplicaron la metodología PEF dentro de las instalaciones de una empresa de partes automotrices donde cal cularon los costos de no calidad.

Tomando como base la metodología PEF, se logró calcular el impacto de cada uno de los riesgos provenientes de los 28 procedimientos estandarizados. Este punto es el factor diferenciador entrela investigación que se tomó como fundamento teórico y el resultado de esta.

Cabe resaltar, de los 3 riesgos identificados en el análisis de costos, a los cuales se les dio un manejo diferente (Ver tabla VI) se debetener especial cuidado y generar las estrategias necesarias para evitar estos costos de fallo externo, ya que en caso de presentarse alguno de ellos, esto sería un riesgo grave para la entidad.

En resumen, vale la pena resaltar, que este trabajo sirve como fundamento teórico para futuras investigaciones que aborden el marco teórico de la estandarización de procesos en Calidad, Seguridad del Paciente o SARLAFT. También, sirve de guía para aquellos investigadores que quieran conocer sobre la aplicación del modelo PEF en una entidad oncológica. Finalmente, este trabajo sirve como un aporte documental quese brinda al sector dela salud, el cual realiza muchas investigaciones, que pocas veces han sido publicadas. conclusiones y recomendaciones. 


\section{Recomendaciones}

Dentro del desarrollo de la presente propuesta, se recomienda a la institución, hacer un Benchmarking con los expertos consultados en la metodología de tiempos sobre aquellas actividades que de la aplicación de este objetivo resultaron con un tiempo mayor al de los expertos.

De acuerdo a los resultados obtenidos en el estudio de costos, se recomienda a la entidad, mantener y/ o aumentar la inversión que tiene en actividades de prevención y evaluación de costos con el propósito de mantener la calidad en la institución y evitar ciertos riesgos provenientes de los costos de no calidad.

Los tiempos en las actividades relacionadas con seguimiento y control que presenta la institución frente al patrón estándar suelen estar por encima en la mayoría de los procedimientos, por lo que se recomienda la implementación deun softwareque facilite y optimice estos procesos.

\section{Vil. Conclusiones}

La propuesta deestandarización planteada permitió aplicar herramientas deingeniería en el área de Calidad, Seguridad del Paciente y SARLAFT que facilitará la toma de decisiones y optimice la gestión de las diferentes actividades realizadas a través de un sistema degestión documental actualizado y respaldado por indicadores de gestión.

En lo referente al estudio de tiempos al clasificar las actividades del área en rutinarias y no rutinarias permitió considerar la opinión de varios expertos en los temas propuestos, además de establecer un patrón de comparación con el fin de que la institución evalué sus procesos frente a otras instituciones y asimismo identificar estrategias de mejora en los puntos críticos detectados.

En cuanto a la metodología PEF se detectó la importancia deevaluar las actividades relacionadas a los riesgos con el fin de identificar cuales operaciones de prevención o evaluación pueden afectar de manera indirecta a más de un riesgo, lo que disminuiría considerablementelos costos totales, ya que los costos por profesional involucrado no se duplicarían reduciendo los costos por actores.
Asimismo, la implementación de actividades orientadas a la prevención y seguimiento, ya que la inversión en estás reduce directamente a la posibilidad de que se presenten fallas, lo que a su vez se ve reflejado en el costo.

Por último, vale resaltar el desarrollo de una propuesta integral basada en la aplicación de herramientas de ingeniería que permitiera optimizar los métodos de trabajo del área además de detectar actividades que no generan valor agregado a su cadena. Asimismo, propiciar un modelo de estandarización documental al ineado a los objetivos institucionales que permita la articulación de los procesos de la institución enfocado en la autoevaluación y mejora continua.

\section{Referencias}

[1] Organización Panamericana de la Salud- OPS, Calidad delos Servicios deSalud en A mérica Latinay el Caribe: Desafíos parala enfermería. En Programa de Organización y Gestión de Sistema de Servicios de Salud. HSO. 2001.

[2] A. Donabedian, Calidad de la atención en salud. Vol. 3 № 1 y 2. México D.F. 1996.

[3] V. González, J. Valecillos \& C. Hernández, Calidad en la prestación de servicios de salud: Parámetros de medición. Revista de Ciencias Sociales (Ve), 19(4), 663-671. 2013.

[4] J. Nuñez, H. Mora \& A . Á vila, Implementación del sistema Sarlaft en los agentes de seguridad social en Salud. Documentos DeTrabajo A reandina, (1). https:/ / doi.org/ 10.33132/ 26654644.1457W.-K 2018.

[5] M. Pérez, Estandarización de procesos de la Empresa Textiles Técnicos (Bachelor's thesis, Universidad Técnica de A mbato. Facultad delngeniería en Sistemas, Electrónica e Industrial. Carrera de Ingeniería Industrial en Procesos de Automatización). 2014.

[6] A. Franco, La seguridad clínica de los pacientes: entendiendo el problema. Colombia Médica, 36(2), 130-133. 2005.

[7] A.Thompson y K. Strikland, Dirección y administración estratégicas. Conceptos, casos y lecturas. México: MacGraw-Hill Interamericana. 1998.

[8] D. Betancourt, Matriz devester para la priorización de problemas. Recuperado el 16 deenero de2020, delngenio Empresa: www.ingenioempresa.com/ matriz-de-vester. 2016.

Rev. Ingeniería, M atemáticas y Ciencias de la Información Vol. 7 / Núm. 14 / julio - diciembre de 2020; pág. 39-57 
[9] M. García Ferrando, La Encuesta. En M. García Ferrando, J. Ibáñez y F. Alvira (Comp.), El análisis dela real idad social. Métodos y técnicas deinvestigación (pp. 123-152). Madrid, España: Alianza Universidad. 1993.

[10] K. Ishikawa, Diagrama delshikawa. Instituto para el aseguramiento dela calidad. 2013.

[11] R. Criollo \& J. Magaña, Estudio del trabajo. McGraw Hill. 2005.

[12] Universidad Nacional, "Guía metodológica para el estudio de cargas de trabajo 2013". pp. 1-10, 2013.

[13] D. Velimirovića, M. Velimirovićb, R. Stankovića, Roleand importanceof key performanceindicators measurement Recovered from: http:/ / www. sjm06.com/ SJM \%20ISSN 1452-4864/ 6_1_2011_ May_1-121/6_1_63-72.pdf. 2011.
[14] S. Gracia \& D. López, M odelo PEF de costes de la calidad como herramienta degestión en empresas constructoras: una visión actual. Revista ingeniería de Construcción, 22 (1), 43-56. 2007.

[15] K. Tapia \& J. Rodolfo, Un sistema de costos decalidad piloto aplicando el model o PEF en la empresa FlexN GateHermosillo (Master's thesis, Keith Tapia, Jesús Rodolfo). 2013.

[16] Instituto Colombiano de Normas Técnicas y Certificación, (2011)" NTC-ISO 31000".

[17] A. Feighbaum, “Total Quality Control”, McGraw Hill Editores. 1991.

[18] R. Hernández, "M etodología dela investigación". México, McGraw-Hill Editores, pp. 453-455. 1998. 
